\title{
Fracturas de acetábulo en la población mexicana
}

\author{
Jorge Zyman Corenstein,* Antonio Martínez Del Campo Sánchez** \\ Unidad Médica de Alta Especialidad del Hospital de Traumatología y Ortopedia «Lomas Verdes»
}

RESUMEN. Introducción: Las fracturas acetabulares cada día se ven más frecuentemente en los servicios de urgencia, aunque a comparación con otras fracturas, son lesiones bastante raras; es de suma importancia la comprensión y adecuado conocimiento para los traumatólogos en urgencias. El determinar la prevalencia de fracturas de acetábulo, así como las características de los pacientes, mecanismo de lesión y lesiones asociadas, nos permitirá desarrollar y mejorar las estrategias de diagnóstico y tratamiento para estos pacientes, así como futuras investigaciones terapéuticas. Material y métodos: Diseño transversal, descriptivo y retrospectivo. Se revisaron los casos con fracturas de acetábulo tratados durante el 1 de Noviembre de 2012 al 31 de Octubre de 2016. Resultados: La mayoría de los pacientes fueron hombres (75\%). Los tipos de fracturas más frecuentes fueron la de pared posterior (22.3\%), seguidas de ambas columnas (19.0\%). El mecanismo de la lesión fue diferente por género, el choque automovilístico es 1.1 veces más probable en la mujeres que en los hombres, pero la caída desde tres 0 más metros es 6.0 veces más probable en los hombres; las mujeres en cambio, tienen 1.9 veces más probabilidad de ser atropelladas y 2.0 veces de sufrir caída desde su plano de sustentación $(p=0.02)$. Las fracturas con mayor proporción de lesiones ortopédicas asociadas corresponden en primer lugar a las de pared anterior de las cuales $\mathbf{8 0 . 0} \%$ tuvo lesiones asociadas. Discusión: Nuestros resultados concuerdan con lo reportado en la literatura a nivel internacional.

Palabras clave: Fracturas, acetábulo, prevalencia, México, diagnóstico.
ABSTRACT. Introduction: Acetabular fractures are seen more frequently in emergency services, although compared to other fractures, they are quite rare injuries; it is of paramount importance the understanding and adequate knowledge for the orthopedic surgeons. Determining the prevalence of acetabulum fractures, as well as patient characteristics, injury mechanism and associated injuries; it will allow us to develop and improve the diagnostic and treatment strategies for these patients, as well as future therapeutic research in our hospital. Material and methods: Transversal design, descriptive and retrospective. We reviewed cases with acetabulum fractures treated during November 1, 2012 to October 31, 2016. Results: Most of the patients were men $(75 \%)$. The most common types of fractures were posterior wall $(22.3 \%)$, followed by both columns $(\mathbf{1 9 . 0} \%)$. The mechanism of the lesion was different by gender, the automobile accident is $\mathbf{1 . 1}$ times more likely in women than in men, but the fall from three or more meters is 6.0 times more likely in men; instead, women are 1.9 times more likely to be hit and 2.0 times to fall from their supportive plane (p $=0.02$ ). The fractures with the highest proportion of associated orthopedic injuries correspond first to those of anterior wall of which $80.0 \%$ had associated injuries. Discussion: Our results are consistent with what is reported in the literature at the international level.

Key words: Fracture, acetabullum, prevalence, Mexico, diagnostic.

Nivel de evidencia: IV

* Médico adscrito al Servicio de Ortopedia y Traumatología, Hospital Ángeles Lomas.

** Médico adscrito, Unidad Médica de Alta Especialidad del Hospital de Traumatología y Ortopedia «Lomas Verdes».

Dirección para correspondencia:

Dr. Jorge Zyman Corenstein

Hospital Ángeles Lomas

Av. Vialidad de la Barranca S/N, consultorio 135, Col. Valle de las Palmas, CP 53763, Huixquilucan, Estado de México. Teléfono: $5531-5482$

E-mail: dr.jorgezyman@gmail.com

Este artículo puede ser consultado en versión completa en http://www.medigraphic.com/actaortopedica 


\section{Introducción}

Las fracturas acetabulares cada día se ven más con más frecuencia en los servicios de urgencia, aunque, a comparación con otras fracturas, son lesiones bastante raras, con una incidencia de tres por cada 100,000 anualmente ${ }^{1}$ y constituyen $0.3-6 \%$ de todas las fracturas; ${ }^{2}$ es de suma importancia la comprensión y adecuado conocimiento para los traumatólogos en urgencias. La mayoría de estas lesiones ocurre en una distribución de edad bimodal: los adultos jóvenes más frecuentemente sufriendo lesiones con mecanismo de alta energía (por ejemplo, accidentes automovilísticos y caídas de alturas) y los ancianos sostienen mecanismos de lesiones de baja energía (por ejemplo, caída desde su plano de sustentación). ${ }^{3}$

Las fracturas acetabulares con frecuencia resultan de traumatismos indirectos, transmitidos a través del fémur, después de un golpe al trocánter mayor, una rodilla flexionada o a través del pie con la rodilla extendida. Existe, por lo tanto, una asociación frecuente con fracturas de la extremidad inferior. ${ }^{4}$

Conocer el tipo o clasificación de la fractura es de suma importancia; no existe un abordaje único para obtener un acceso conveniente a ambas columnas en el caso de que requiera tratamiento quirúrgico, ${ }^{5}$ por ello conocer el trazo de la fractura y su clasificación ayuda para la planificación quirúrgica y así escoger un abordaje conveniente. También es de suma importancia conocer las lesiones a las que se asocian este tipo de fracturas.

Para la evaluación de una fractura de acetábulo, se debe comenzar con radiografías anteroposteriores (AP) de pelvis y proyecciones oblicuas de Judet (obturador y alar) y después una tomografía axial computarizada (TAC). ${ }^{2,6}$ Está descrito que la TAC aumenta la confiabilidad interobservador de la clasificación, comparado con las radiografías. ${ }^{7}$

En la actualidad se utilizan principalmente dos sistemas de clasificación, la clasificación de Judet y Letournel y la clasificación AO.

El sistema de clasificación de fractura de acetábulo de Judet y Letournel está basado en el concepto anatómico de que el acetábulo está compuesto por dos columnas; divide las lesiones en 10 patrones, cinco elementales y cinco asociadas, basadas en hallazgos quirúrgicos y radiografías características. También proporciona la descripción fundamental de las dos columnas del acetábulo. ${ }^{6,8,9,10}$

La clasificación AO incorpora el concepto de la clasificación de Letournel y divide a las fracturas en A, B o $\mathrm{C}$, dependiendo de si el trazo de la fractura involucra una columna, ambas columnas o si existe un trazo transverso; posteriormente se sigue subdividiendo dependiendo de la variación de la fractura. , $^{81}$

La literatura mundial sobre la epidemiología de las fracturas de acetábulo es muy escasa y en nuestro país, según nuestro conocimiento, no existe algún estudio que describa la epidemiología de las fracturas de acetábulo. ${ }^{12}$

El propósito de este estudio es determinar la prevalencia de fracturas de acetábulo, así como las características de los pacientes, mecanismo de lesión y lesiones asociadas; este conocimiento nos permitirá desarrollar y mejorar las estrategias de diagnóstico y tratamiento para estos pacientes, así como futuras investigaciones.

\section{Material y métodos}

Diseño del estudio: transversal, descriptivo y retrospectivo. Período de estudio: del 1 de Noviembre de 2012 al 31 de Octubre de 2016.

Criterios de inclusión: 1) pacientes con fractura de acetábulo, 2) cualquier sexo, 3) mayores de 16 años.

Criterios de exclusión y de eliminación: 1) expediente incompleto.

Se identificó en la bitácora de registros de pacientes del servicio a los casos que cumplieran con los criterios de selección. Las variables que se registraron fueron el sexo, la edad, clasificación de la fractura de acetábulo, lesiones ortopédicas asociadas y el mecanismo de la lesión. Posteriormente, se realizó la captura de la información en una base de datos creada para el presente estudio en el programa Microsoft Excel y se analizó estadísticamente utilizando el programa SPSS (paquete estadístico para las ciencias sociales).

Aspectos éticos. De acuerdo con la Ley General de Salud Vigente en Materia de Investigación el presente estudio fue clasificado como un estudio sin riesgo, ya que la información se recabó de manera retrospectiva.

\section{Resultados}

Se revisaron 184 casos de fracturas de acetábulo distribuidos en 138 hombres (75\%) y 46 mujeres (25\%) con una edad promedio de 42.9 años \pm 18.9 de desviación estándar (rango 16-91 años). No hubo una diferencia significativa en las medias de la edad entre hombres y mujeres con $42.1 \pm$ 18.0 para los primeros versus $44.3 \pm 21.7$ para las segundas $(\mathrm{p}=0.54)$.

De acuerdo con la clasificación de Judet y Letournel, los tipos de fracturas más frecuentes fueron de pared posterior (22.3\%), seguidas de ambas columnas (19.0\%), transversas (15.8\%) y, en cuarto lugar, la T (10.9\%); como se observa en la Figura 1, las fracturas de columna anterior fueron las de menor frecuencia con $2.2 \%$.

El choque de automóvil constituyó el mecanismo de lesión más frecuente con $41.3 \%$ de los casos y le siguió en segundo lugar las caídas de tres o más metros (20.7\%), en tercer lugar, el choque de motocicletas (10.9\%) y en cuarto lugar los atropellamientos con 9.8\% de los casos (Figura 2).

Un poco más de la mitad (56.5\%) tuvo lesiones asociadas, siendo la luxación de cadera aislada la más común en 7.1\% de los casos.

Las lesiones más frecuentes ocasionadas por choque de automóvil se caracterizaron por fracturas de pared posterior en $34.2 \%$ de los casos, transversas en $15.8 \%$, de columna hemitransversa posterior en $10.5 \%$ y de ambas columnas 

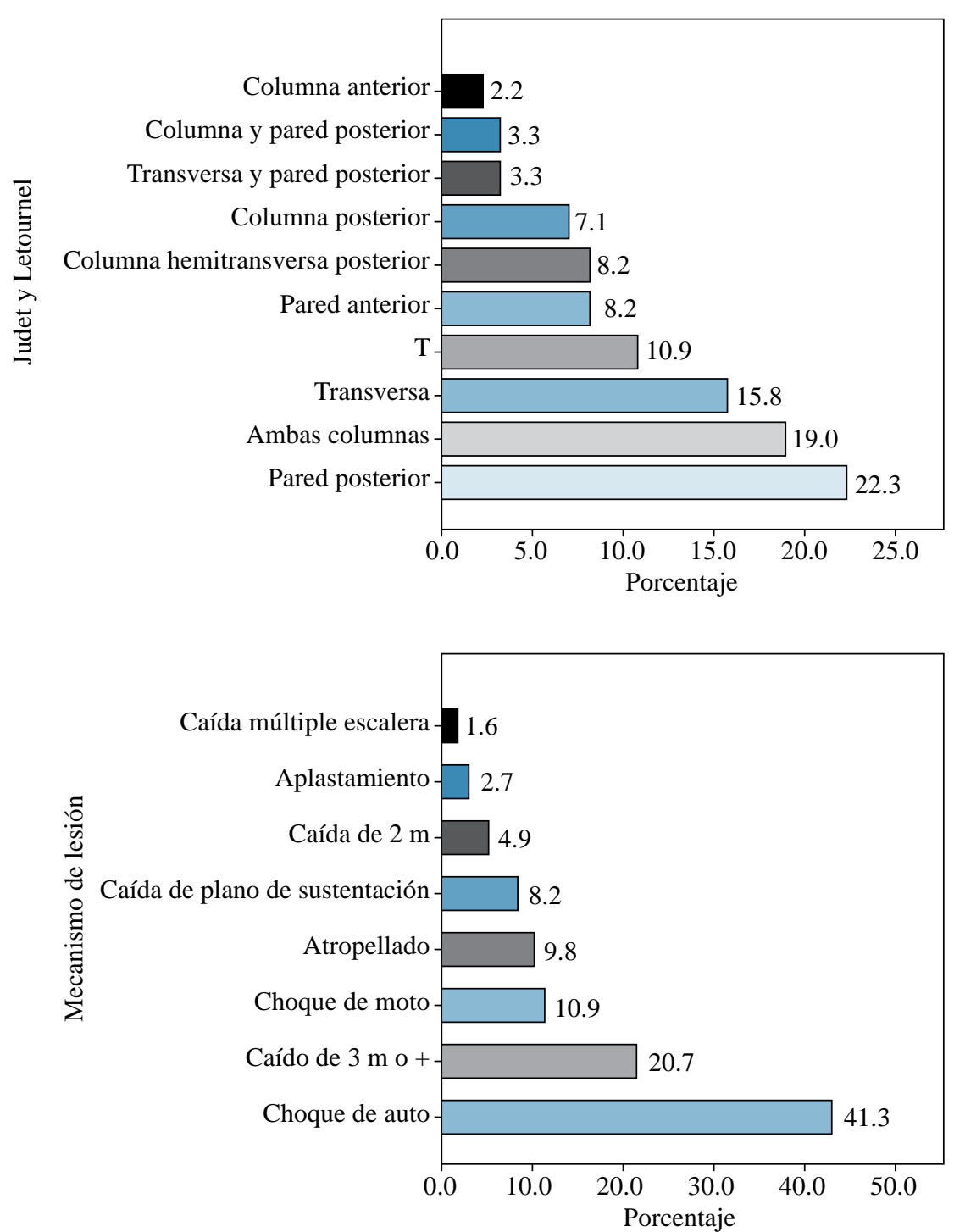

Figura 1:

Porcentajes de los tipos de fracturas de acetábulo de acuerdo con la escala de Judet y Letournel (n $=184$ ).

Figura 2:

Porcentajes de los mecanismos de lesión de las fracturas de acetábulo $(n=184)$. en 9.2\%. Por caída de tres o más metros produjeron en primer lugar fracturas de ambas columna $42.1 \%$, en $\mathrm{T} 13.2 \%$, transversas $20.0 \%$ y de pared anterior $10.5 \%$. Por choque de moto en primer lugar fracturas de pared posterior $35.0 \%$ y en segundo las transversas en $20.0 \%$ (estas lesiones ocupan en mismo lugar por choque de auto), en tercer lugar los choques de moto produjeron fracturas de ambas columnas $15.0 \%$ y de columna posterior en cuarto con $10.0 \%$ de los casos. Los atropellamientos se caracterizaron por fracturas $\mathrm{T}$ en $22.2 \%$; y con $16.7 \%$ para cada una: pared posterior, transversas y de pared anterior. Las caídas desde el propio plano de sustentación ocasionaron fracturas de ambas columnas $33.3 \%$, transversas $20.0 \%$, pared anterior $13.3 \%$ y columna anterior $13.3 \%$. Por último, otros mecanismos conjuntos ocasionaron fracturas transversas, $\mathrm{T}$ y de pared anterior en $16.7 \%$, respectivamente, y de pared posterior en $11.8 \%(\mathrm{p}=0.01)$ (Figura 3).

Por otra parte, el mecanismo de la lesión fue diferente por género (Tabla 1) ya que el choque de auto es 1.1 veces más probable en la mujeres que en los hombres; pero la caída desde tres o más metros es 6.0 veces más probable en los hombres y 1.3 veces en éstos el choque de moto; las mujeres, en cambio, tienen 1.9 veces más probabilidad de ser atropelladas y 2.0 veces de sufrir caída desde su plano de sustentación ( $\mathrm{p}=0.02)$.

La edad también es diferente según el mecanismo de la lesión (Tabla 2). La edad promedio más baja corresponde a los choques de moto, siendo ligeramente menor en las mujeres (27.5 años versus 31.9 de los hombres); la siguiente edad corresponde al choque de auto con una edad promedio ligeramente mayor en las mujeres (36.4 años versus 34.9 de los hombres); la edad promedio más elevada corresponde a la caída desde el plano de sustentación prácticamente igual por género con 75.4 de los hombres versus 74.6 de las mujeres (para la diferencia de edad en general según mecanismo de lesión $\mathrm{p}=0.0001$; edad entre hombres y mujeres $\mathrm{p}=$ $0.83)$.

Las fracturas con mayor proporción de lesiones ortopédicas asociadas corresponden en primer lugar a las de pared anterior, de las cuales $80.0 \%$ tuvo lesiones asociadas; en se- 


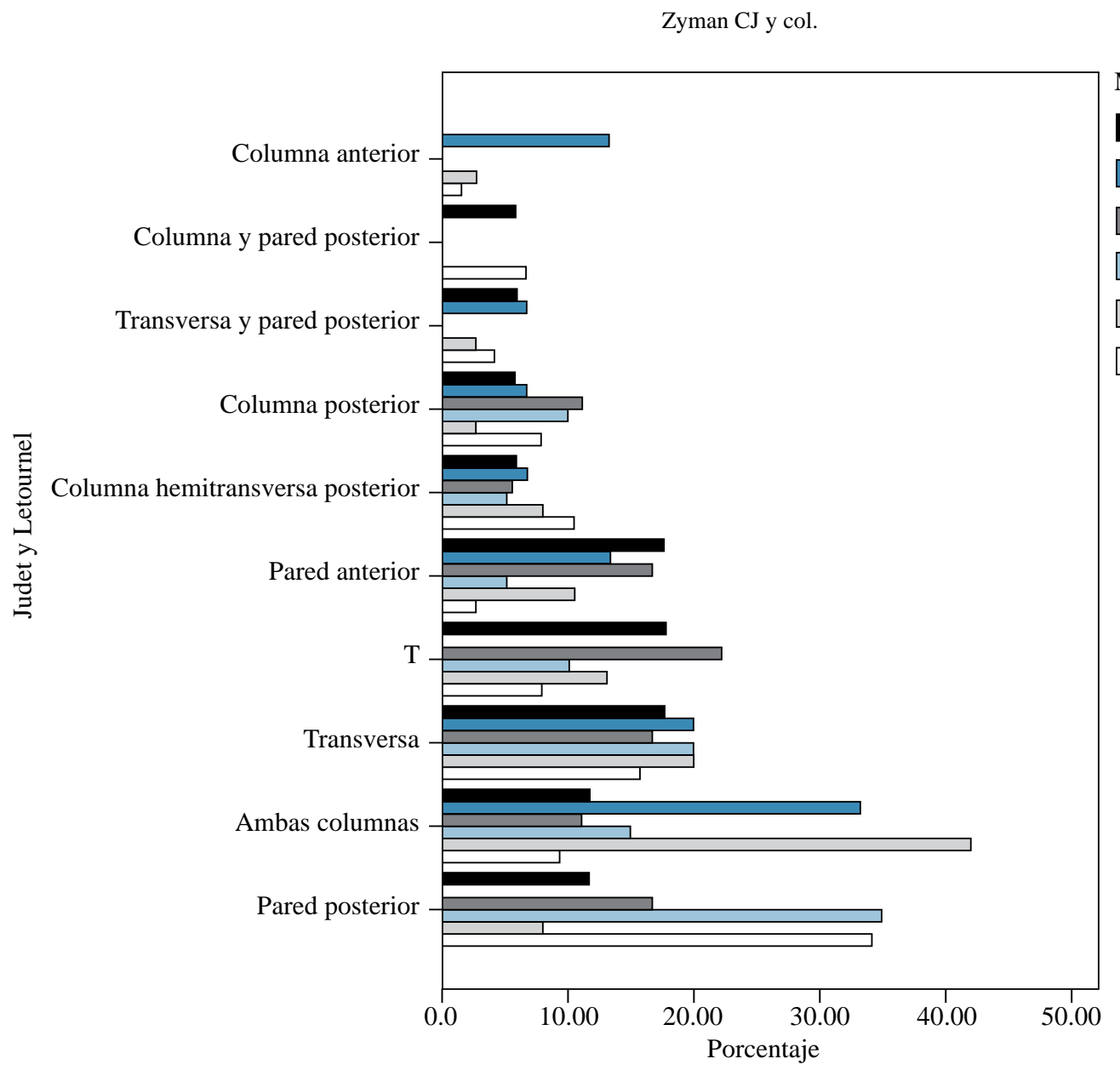

Mecanismo de lesión

Otros

Caída de plano de sustentación

Atropellado

Choque de moto

Caída de 3 m o +

Choque de auto

Figura 3:

Asociación entre tipos de fracturas de acetábulo (clasificación de Judet y Letournel) y mecanismo de lesión $(\mathrm{n}=184)$.

\begin{tabular}{|c|c|c|c|c|c|}
\hline & & & \multicolumn{2}{|c|}{ Género } & \multirow[b]{2}{*}{ Total } \\
\hline & & & Femenino & Masculino & \\
\hline \multirow[t]{12}{*}{$\begin{array}{l}\text { Mecanismo } \\
\text { de lesión }\end{array}$} & Choque de auto & $\mathrm{n}$ & 21 & 55 & 76 \\
\hline & & $\%$ & 45.7 & 39.9 & 41.3 \\
\hline & Caída de $3 \mathrm{mo}+$ & $\mathrm{n}$ & 2 & 36 & 38 \\
\hline & & $\%$ & 4.3 & 26.1 & 20.7 \\
\hline & Choque de moto & $\mathrm{n}$ & 4 & 16 & 20 \\
\hline & & $\%$ & 8.7 & 11.6 & 10.9 \\
\hline & Atropellado & $\mathrm{n}$ & 7 & 11 & 18 \\
\hline & & $\%$ & 15.2 & 8.0 & 9.8 \\
\hline & Caída de plano de sust. & $\mathrm{n}$ & 6 & 9 & 15 \\
\hline & & $\%$ & 13.0 & 6.5 & 8.2 \\
\hline & Otros & $\mathrm{n}$ & 6 & 11 & 17 \\
\hline & & $\%$ & 13.0 & 8.0 & 9.2 \\
\hline \multirow[t]{2}{*}{ Total } & & $\%$ & 46 & 138 & 184 \\
\hline & & $\mathrm{n}$ & 100.0 & 100.0 & 100.0 \\
\hline
\end{tabular}

gundo lugar, las T (75\%); en tercer lugar, las de columna hemitransversa posterior (73.3\%); en cuarto lugar, las transversas (55.2\%) y en quinto las de pared posterior (53.7\%). La relación entre tipo de fractura y lesiones asociadas arroja una $\mathrm{p}=0.03$. Al mismo tiempo, las lesiones asociadas fueron proporcionalmente más altas en las mujeres, ya que de

\begin{tabular}{llllll}
\multicolumn{2}{|c}{ Tabla 2: Asociación entre edad por género y mecanismo de } \\
lesión de las fracturas de acetábulo.
\end{tabular}

46 de ellas hasta 65.2\% tuvieron algún tipo de lesión asociada para un riesgo relativo de 1.4 [IC 95\% 0.8-2.4] veces más que los hombres, ya que de 138 de ellos 53.3\% tuvo alguna lesión asociada ( $\mathrm{p}=0.11)$.

No se observa diferencia en el tipo de fractura y el género de los casos $(\mathrm{p}=0.90)$. Sin embargo, la edad sí es muy diferente ( $p=0.0001)$, siendo la más alta con 56.0 años pro- 
Tabla 3: Medias de edad según tipo de fracturas de acetábulo.

$\begin{array}{lrrrr} & & & \text { Desviación } \\ & \mathrm{n} & \text { Media } & \text { típica } & \mathrm{p} \\ & & & & \\ \text { Pared posterior } & 41 & 33.54 & 13.073 & \\ \text { Ambas columnas } & 35 & 49.63 & 17.876 & \\ \text { Transversa } & 29 & 41.21 & 21.333 & \\ \text { T } & 20 & 40.00 & 19.585 & \\ \text { Pared anterior } & 15 & 55.87 & 20.308 & 0.0001 \\ \text { Columna hemitransversa } & 15 & 44.20 & 17.367 & \\ \text { posterior } & & & & \\ \text { Columna posterior } & 13 & 33.62 & 16.914 & \\ \text { Transversa y pared } & 6 & 49.33 & 17.154 & \\ \text { posterior } & & & & \\ \text { Columna y pared } & 6 & 54.67 & 17.671 & \\ \text { posterior } & & & & \\ \text { Columna anterior } & 4 & 56.00 & 16.833 & \\ \text { Total } & 184 & 42.90 & 18.978 & \end{array}$

medio las de columna anterior y la más baja edad corresponde a las de pared posterior con 33.5 años en promedio (Tabla 3). A partir de la mediana (39 años) se forman dos grupos característicos de los tipos de fractura, así para los que están debajo de 39 años en primer lugar están las fracturas de columna posterior, en segundo las de pared posterior, en tercero las transversa y en cuarto la T; en cambio, en los pacientes por arriba de 39 años en primer lugar están las fracturas de columna y pared posterior, en segundo columna anterior, en tercero pared anterior y en cuarto lugar ambas columnas $(\mathrm{p}=0.001)$.

\section{Discusión}

El objetivo de este estudio es presentar nuestra experiencia de pacientes con fractura del acetábulo; para esto se necesita un adecuado diagnóstico mediante un estudio radiográfico que incluye radiografías de pelvis anteroposterior y proyecciones oblicuas de Judet (obturador y alar), así como una tomografía axial computarizada, esto con el fin de poder llevar a cabo una adecuada clasificación de la fractura y una decisión en cuanto al tratamiento, en caso de que requiera cirugía, la clasificación nos ayuda a decidir una vía de abordaje.

Nuestro total de pacientes fue de 184; 75\% de los cuales fueron pacientes del sexo masculino. Esto llama mucho la atención cuando se describe el mecanismo de la lesión; un choque automovilístico es más probable en las mujeres que en los hombres, pero la caída desde tres o más metros es 6.0 veces más probable en los hombres y 1.3 veces en éstos el choque de moto; las mujeres en cambio, tienen 1.9 veces más probabilidad de ser atropelladas y 2.0 veces de sufrir caída desde su plano de sustentación ( $\mathrm{p}=0.02)$.

Las fracturas con mayor proporción de lesiones asociadas corresponden en primer lugar a las de pared anterior; de las cuales, $80.0 \%$ tuvo lesiones asociadas, en segundo lugar las T (75\%), en tercer lugar las de columna hemitransversa posterior (73.3\%), en cuarto lugar las transversas (55.2\%) y en quinto las de pared posterior (53.7\%). La relación entre tipo de fractura y lesiones asociadas arroja una $\mathrm{p}=0.03$; al mismo tiempo, las lesiones asociadas fueron proporcionalmente más altas en las mujeres ya que, de 46 de ellas, hasta $65.2 \%$ tuvo algún tipo de lesión asociada para un riesgo relativo de 1.4 [IC 95\% 0.8-2.4] veces más que los hombres ya que de 138 de ellos 53.3\% tuvo alguna lesión asociada $(\mathrm{p}=0.11)$.

Liebergall y colaboradores, en $1999,{ }^{1}$ describion un estudio en el que encontraron que pacientes con trazos de fractura simples (lo cuales sólo involucran un componente) tienen mejores resultados que pacientes con fracturas más complejas; concluyeron que esto es lógico porque un solo componente puede manejarse más fácil que múltiples fragmentos.

Matta $^{6}$ en 1996 realizó un estudio de 259 pacientes en los cuales según la clasificación de Judet y Letournel las fracturas asociadas fueron 208 (79\%) siendo la fractura de ambas columnas la más común (35\%).

Mesbahi y colaboradores, ${ }^{13,14}$ en el 2017, describieron un estudio de 163 pacientes con edad media de 36.84 años; 82.3\% de sus pacientes con fractura de acetábulo fueron causa de una accidente automovilístico y $15.2 \%$ por una caída de altura. El trazo de fractura más común fue el de columna posterior en $43 \%$ de los casos y ambas columna es $21.5 \%$ de los pacientes. En nuestro estudio, de acuerdo con la clasificación de Judet y Letournel, los tipos de fracturas más frecuentes fueron la de pared posterior (22.3\%), seguidas de ambas columnas (19.0\%).

Una de las limitaciones del presente estudio, y que es propósito de una nueva investigación, es el pronóstico y resultados terapéuticos, no considerados en este trabajo.

Nuestros resultados concuerdan con lo reportado en la literatura que revisamos, en cuanto a las características epidemiológicas. Éste es el primer estudio en nuestro hospital que analiza las características antes mencionadas en fracturas de acetábulo.

Bibliografía

1. Mauffrey C, Hao J, Cuellar DO 3rd, Herbert B, Chen X, Liu B, et al. The epidemiology and injury patterns of acetabular fractures: are the USA and China comparable? Clin Orthop Relat Res. 2014; 472(11): 3332-7.

2. Estrems-Díaz V, Hernández-Ferrando L, Balaguer-Andrés J, BruPomer A. Acetabular fractures: short-term results. Rev Esp Cir Ortop Traumatol. 2012; 56(1): 17-23.

3. Liebergall M, Mosheiff R, Low J, Goldvirt M, Matan Y, Segal D. Acetabular fractures. Clinical outcome of surgical treatment. Clin Orthop Relat Res. 1999; (366): 205-16.

4. Helfet DL, Bartlett II CS. Acetabular fractures Evaluation/ classification/treatment concepts and approaches in AO principles of fracture management. Stuttgart, New York: Thieme; 2000: 415-438.

5. Alton TB, Gee AO. Classifications in brief: Letournel classification for acetabular fractures. Clin Orthop Relat Res. 2014; 472(1): 35-8.

6. Lawrence DA, Menn K, Baumgaertner M, Haims AH. Acetabular fractures: anatomic and clinical considerations. AJR Am J Roentgenol. 2013; 201(3): W425-36.

7. Ohashi K, El-Khoury GY, Abu-Zahra KW, Berbaum KS. Interobserver agreement for Letournel acetabular fracture classification with multidetector CT: are standard Judet radiographs necessary? Radiology. 2006; 241(2): 386-91. 
8. Letournel E. Acetabulum fractures: classification and management. Clin Orthop Relat Res. 1980; (151): 81-106.

9. Judet R, Judet J, Letournel E. Fractures of the acetabulum: classification and surgical approaches for open reduction. Preliminary report. J Bone Joint Surg Am. 1964; 46: 1615-46.

10. Letournel E, Judet R. Fractures of the acetabulum. 2nd ed. In: Elson RA, transl, ed. New York, NY: Springer; 1993.

11. Marsh JL, Slongo TF, Agel J, Broderick JS, Creevey W, DeCoster TA, et al. Fracture and dislocation classification compendium 2007: Orthopaedic Trauma Association classification, database and outcomes committee. J Orthop Trauma. 2007; 21(10 Suppl): S1133.

12. Laird A, Keating JF. Acetabular fractures: a 16-year prospective epidemiological study. J Bone Joint Surg Br. 2005; 87(7): 969-73.

13. Matta JM. Fractures of the acetabulum: accuracy of reduction and clinical results in patients managed operatively within three weeks after the injury. J Bone Joint Surg Am. 1996; 78(11): 1632-45.

14. Mesbahi SAR, Ghaemmaghami A, Ghaemmaghami S, Farhadi P. Outcome after surgical management of acetabular fractures: a 7-year experience. Bull Emerg Trauma. 2018; 6(1): 37-44. 